\title{
INDEX TRANSFORMS WITH THE PRODUCT OF THE ASSOCIATED LEGENDRE FUNCTIONS
}

\author{
S. YAKUBOVICH
}

\begin{abstract}
Index transforms with the product of the associated Legendre functions are introduced. Mapping properties are investigated in the Lebesgue spaces. Inversion formulas are proved. The results are applied to solve a boundary value problem in a wedge for a third order partial differential equation.
\end{abstract}

Keywords : Index Transforms, Associated Legendre functions, modified Bessel functions, Fourier transform, Mellin transform, Boundary value problem

MS Classification: 44A15, 33C10, 33C45, 44A05

\section{INTRODUCTION AND PRELIMINARY RESULTS}

Let $\mu \in \mathbb{C}, f(x), g(\tau), x, \tau \in \mathbb{R}_{+}$be complex-valued functions. In the present paper we will investigate mapping properties of the following index transforms [1]

$$
\begin{gathered}
F(\tau)=\sqrt{\pi} \Gamma(1+i \tau-\mu) \Gamma(1-i \tau-\mu) \int_{0}^{\infty} P_{i \tau}^{\mu}(\sqrt{1+x}) P_{-i \tau}^{\mu}(\sqrt{1+x}) \\
\times \frac{f(x)}{\sqrt{1+x}} d x \\
G(x)=\sqrt{\frac{\pi}{1+x}} \int_{0}^{\infty} \Gamma(1+i \tau-\mu) \Gamma(1-i \tau-\mu) P_{i \tau}^{\mu}(\sqrt{1+x}) P_{-i \tau}^{\mu}(\sqrt{1+x}) \\
\times g(\tau) d \tau .
\end{gathered}
$$

Here $i$ is the imaginary unit, $\Gamma(z)$ is the Euler gamma-function and $P_{\nu}^{\mu}(z)$ is the associated Legendre function of the first kind (see [2], Vol. I, 33). Our goal is to study them mapping properties, prove inversion theorems and apply to solve a boundary value problem for a higher order PDE. Denoting the kernel of (1.1), (1.2) by 
$\Phi(x, \tau)=\sqrt{\frac{\pi}{1+x}} \Gamma(1+i \tau-\mu) \Gamma(1-i \tau-\mu) P_{i \tau}^{\mu}(\sqrt{1+x}) P_{-i \tau}^{\mu}(\sqrt{1+x})$,

we will find for further use its representation in terms of Fourier cosine transform [6] and deduce an ordinary differential equation with polynomial coefficients, whose solution is $\Phi(x, \tau)$, employing the socalled method of the Mellin-Barnes integrals, which is already being successfully applied by the author for other index transforms. In fact, appealing to [4], Vol. III, entry 8.4.41.50, we find the following MellinBarnes integral representation for the kernel (1.3), namely,

$\Phi(x, \tau)=\frac{1}{2 \pi i} \int_{\gamma-i \infty}^{\gamma+i \infty} \frac{\Gamma(1-s+i \tau) \Gamma(1-s-i \tau) \Gamma(1 / 2-s) \Gamma(s-\mu)}{\Gamma(1-s) \Gamma(1-s-\mu)} x^{-s} d s, x>0$

where $\gamma$ is taken from the interval $(\operatorname{Re} \mu, 1 / 2)$. The absolute convergence of the integral (1.4) follows immediately from the Stirling asymptotic formula for the gamma- function [2], Vol. I, because for all $\tau \in \mathbb{R}$

$$
\begin{gathered}
\frac{\Gamma(1-s+i \tau) \Gamma(1-s-i \tau) \Gamma(1 / 2-s) \Gamma(s-\mu)}{\Gamma(1-s) \Gamma(1-s-\mu)} \\
=O\left(e^{-\pi|s|}|s|^{-1 / 2}\right),|s| \rightarrow \infty .
\end{gathered}
$$

Moreover, it can be differentiated under the integral sign any number of times due to the absolute and uniform convergence by $x \geq x_{0}>0$. Our method of investigation of the index transforms (1.1), (1.2) is based on the Mellin transform technique developed in [5]. Precisely, the Mellin transform is defined, for instance, in $L_{\nu, p}\left(\mathbb{R}_{+}\right), 1 \leq p \leq 2$ (see details in [6]) by the integral

$$
f^{*}(s)=\int_{0}^{\infty} f(x) x^{s-1} d x
$$

being convergent in mean with respect to the norm in $L_{q}(\nu-i \infty, \nu+$ $i \infty), \nu \in \mathbb{R}, q=p /(p-1)$. Moreover, the Parseval equality holds for $f \in L_{\nu, p}\left(\mathbb{R}_{+}\right), g \in L_{1-\nu, q}\left(\mathbb{R}_{+}\right)$

$$
\int_{0}^{\infty} f(x) g(x) d x=\frac{1}{2 \pi i} \int_{\nu-i \infty}^{\nu+i \infty} f^{*}(s) g^{*}(1-s) d s .
$$

The inverse Mellin transform is given accordingly

$$
f(x)=\frac{1}{2 \pi i} \int_{\nu-i \infty}^{\nu+i \infty} f^{*}(s) x^{-s} d s
$$


where the integral converges in mean with respect to the norm in $L_{\nu, p}\left(\mathbb{R}_{+}\right)$

$$
\|f\|_{\nu, p}=\left(\int_{0}^{\infty}|f(x)|^{p} x^{\nu p-1} d x\right)^{1 / p} .
$$

In particular, letting $\nu=1 / p$ we get the usual space $L_{p}\left(\mathbb{R}_{+}\right)$.

We begin with

Lemma 1. Let $x, \tau \in \mathbb{R}_{+}, \operatorname{Re} \mu<1 / 2$. Then the kernel (1.3) has the following integral representation in terms of Fourier cosine transform of the associated Legendre function

$$
\begin{aligned}
\Phi(x, \tau)= & \Gamma\left(\frac{3}{2}-\mu\right) e^{\pi i \mu} \int_{0}^{\infty} \frac{\cos (\tau u)}{\cosh ^{1 / 2}(u / 2)\left(x+\cosh ^{2}(u / 2)\right)^{3 / 4}} \\
& \times P_{1 / 2}^{\mu}\left(\frac{x+2 \cosh ^{2}(u / 2)}{2 \cosh (u)\left(x+\cosh ^{2}(u / 2)\right)^{1 / 2}}\right) d u .
\end{aligned}
$$

Proof. In fact, appealing to the reciprocal formulae via the Fourier cosine transform (cf. formula (1.104) in [1])

$$
\begin{gathered}
\int_{0}^{\infty} \Gamma(1-s+i \tau) \Gamma(1-s-i \tau) \cos (\tau y) d \tau=\frac{\pi}{2^{2(1-s)}} \frac{\Gamma(2(1-s))}{\cosh ^{2(1-s)}(y / 2)}, \operatorname{Re} s<1, \\
\Gamma(1-s+i \tau) \Gamma(1-s-i \tau)=\frac{\Gamma(2(1-s))}{2^{1-2 s}} \int_{0}^{\infty} \frac{\cos (\tau y)}{\cosh ^{2(1-s)}(y / 2)} d y,
\end{gathered}
$$

we replace the gamma-product $\Gamma(1-s+i \tau) \Gamma(1-s-i \tau)$ in the integral (1.4) by its integral representation (1.12) and change the order of integration via Fubini's theorem. Then, employing the duplication formula for the gamma-function [2], Vol. I, we derive

$$
\begin{gathered}
\Phi(x, \tau)=\frac{1}{2 \pi \sqrt{\pi} i} \int_{0}^{\infty} \frac{\cos (\tau u)}{\cosh ^{2}(u / 2)} \\
\times \int_{\gamma-i \infty}^{\gamma+i \infty} \frac{\Gamma(s-\mu) \Gamma(1 / 2-s) \Gamma(3 / 2-s)}{\Gamma(1-s-\mu)}\left(\frac{x}{\cosh ^{2}(u / 2)}\right)^{-s} d s d u .
\end{gathered}
$$

In the meantime, the inner integral with respect to $s$ can be calculated, employing the Parseval equality (1.7), properties of the Mellin transform (1.6) and relations $(8.4 .3 .1),(8.4 .46 .7)$ in [4], Vol. III. Thus we obtain

$$
\frac{1}{2 \pi i} \int_{\gamma-i \infty}^{\gamma+i \infty} \frac{\Gamma(s-\mu) \Gamma(1 / 2-s) \Gamma(3 / 2-s)}{\Gamma(1-s-\mu)}\left(\frac{x}{\cosh ^{2}(u / 2)}\right)^{-s} d s
$$




$$
=\left(\frac{x}{\cosh ^{2}(u / 2)}\right)^{-\mu} \int_{0}^{\infty} y^{1 / 2-\mu} e^{-\left(x \cosh ^{-2}(u / 2)+1\right) y} \Psi\left(\frac{1}{2}-\mu, 2 ; y\right) d y
$$

where $\Psi(a, b ; z)$ is the Tricomi function [2], Vol. I. But entry 7.11.4.9 in [4], Vol. III permits to express Tricomi's function in (1.14) in terms of the Bateman function $k_{\nu}(z)$ [2], Vol. II, and we have

$$
\begin{aligned}
& \left(\frac{x}{\cosh ^{2}(u / 2)}\right)^{-\mu} \int_{0}^{\infty} y^{1 / 2-\mu} e^{-\left(x \cosh ^{-2}(u / 2)+1\right) y} \Psi\left(\frac{1}{2}-\mu, 2 ; y\right) d y \\
= & \Gamma(3 / 2+\mu)\left(\frac{x}{\cosh ^{2}(u / 2)}\right)^{-\mu} \int_{0}^{\infty} y^{-1 / 2-\mu} e^{-\left(x \cosh ^{-2}(u / 2)+1 / 2\right) y} k_{1+2 \mu}\left(\frac{y}{2}\right) d y .
\end{aligned}
$$

Meanwhile, the latter integral is calculated in [4], Vol. III, entry 2.14.2.4 in terms of the Gauss hypergeometric function [2], Vol. I and it gives after slight simplifications

$$
\begin{aligned}
& \Gamma(3 / 2+\mu)\left(\frac{x}{\cosh ^{2}(u / 2)}\right)^{-\mu} \int_{0}^{\infty} y^{-1 / 2-\mu} e^{-\left(x \cosh ^{-2}(u / 2)+1 / 2\right) y} k_{1+2 \mu}\left(\frac{y}{2}\right) d y \\
& =4^{\mu} \sqrt{\pi}\left(\frac{x}{\cosh ^{2}(u / 2)}\right)^{-\mu} \frac{\Gamma(3 / 2-\mu)}{\Gamma(1-\mu)}{ }_{2} F_{1}\left(\frac{1}{2}-\mu, \frac{3}{2}-\mu ; 1-2 \mu ;-\frac{x}{\cosh ^{2}(u / 2)}\right) .
\end{aligned}
$$

Finally, appealing to relation (7.3.1.70) in [4], Vol. III, we express the value of the Gauss hypergeometric function in terms of the associated Legendre function. Precisely, we find

$$
\begin{aligned}
& 4^{\mu} \sqrt{\pi}\left(\frac{x}{\cosh ^{2}(u / 2)}\right)^{-\mu} \frac{\Gamma(3 / 2-\mu)}{\Gamma(1-\mu)}{ }_{2} F_{1}\left(\frac{1}{2}-\mu, \frac{3}{2}-\mu ; 1-2 \mu ;-\frac{x}{\cosh ^{2}(u / 2)}\right) \\
& =\sqrt{\pi} \Gamma\left(\frac{3}{2}-\mu\right) e^{\pi i \mu}\left(1+x \cosh ^{-2}\left(\frac{u}{2}\right)\right)^{-3 / 4} P_{1 / 2}^{\mu}\left(\frac{x+2 \cosh ^{2}(u)}{2 \cosh (u)\left(x+\cosh ^{2}(u / 2)\right)^{1 / 2}}\right) .
\end{aligned}
$$

Hence substituting this value in the right-hand side of (1.14) and recalling (1.13), we arrive at the formula (1.10), completing the proof of Lemma 1. 
Lemma 2. The function $\Phi(x, \tau),(x, \tau) \in \mathbb{R}_{+} \times \mathbb{R}_{+}$given by formula (1.3) satisfies the following third order differential equation with polynomial coefficients

$$
\begin{gathered}
2 x^{2}(1+x) \frac{d^{3} \Phi}{d x^{3}}+x(11 x+6) \frac{d^{2} \Phi}{d x^{2}} \\
+\left(2\left(1-\mu^{2}\right)+x\left(11+2 \tau^{2}\right)\right) \frac{d \Phi}{d x}+\left(1+\tau^{2}\right) \Phi=0 .
\end{gathered}
$$

Proof. As it was mentioned above, the asymptotic behavior (1.5) of the integrand in (1.4) permits a differentiation under the integral sign any number of times. Hence employing the reduction formula for the gamma- function [2], Vol. I , we derive

$$
\begin{gathered}
\left(x \frac{d}{d x}\right)^{2} \Phi(x, \tau)=\frac{1}{2 \pi i} \int_{\gamma-i \infty}^{\gamma+i \infty} \frac{s^{2} \Gamma(1-s+i \tau) \Gamma(1-s-i \tau) \Gamma(1 / 2-s) \Gamma(s-\mu)}{\Gamma(1-s) \Gamma(1-s-\mu)} x^{-s} d s \\
=\frac{1}{2 \pi i} \int_{\gamma-i \infty}^{\gamma+i \infty} \frac{\Gamma(2-s+i \tau) \Gamma(2-s-i \tau) \Gamma(1 / 2-s) \Gamma(s-\mu)}{\Gamma(1-s) \Gamma(1-s-\mu)} x^{-s} d s \\
-\left(1+\tau^{2}\right) \Phi(x, \tau)-2 x \frac{d \Phi}{d x} .
\end{gathered}
$$

Meanwhile, with a simple change of variable

$$
\begin{aligned}
& \frac{1}{2 \pi i} \int_{\gamma-i \infty}^{\gamma+i \infty} \frac{\Gamma(2-s+i \tau) \Gamma(2-s-i \tau) \Gamma(1 / 2-s) \Gamma(s-\mu)}{\Gamma(1-s) \Gamma(1-s-\mu)} x^{-s} d s \\
= & \frac{1}{2 \pi i} \int_{\gamma-1-i \infty}^{\gamma-1+i \infty} \frac{\Gamma(1-s+i \tau) \Gamma(1-s-i \tau) \Gamma(-1 / 2-s) \Gamma(s+1-\mu)}{\Gamma(-s) \Gamma(-s-\mu)} x^{-s-1} d s \\
= & -\frac{1}{2 \pi i} \int_{\gamma-1-i \infty}^{\gamma-1+i \infty} \frac{s\left(s^{2}-\mu^{2}\right) \Gamma(1-s+i \tau) \Gamma(1-s-i \tau) \Gamma(1 / 2-s) \Gamma(s-\mu)}{(1 / 2+s) \Gamma(1-s) \Gamma(1-s-\mu)} x^{-s-1} d s \\
= & -\frac{1}{2 \pi i} \int_{\gamma-1-i \infty}^{\gamma-1+i \infty} \frac{\left(s^{2}-\mu^{2}\right) \Gamma(1-s+i \tau) \Gamma(1-s-i \tau) \Gamma(1 / 2-s) \Gamma(s-\mu)}{\Gamma(1-s) \Gamma(1-s-\mu)} x^{-s-1} d s \\
+ & \frac{1}{4 \pi i} \int_{\gamma-1-i \infty}^{\gamma-1+i \infty} \frac{\left(s^{2}-\mu^{2}\right) \Gamma(1-s+i \tau) \Gamma(1-s-i \tau) \Gamma(1 / 2-s) \Gamma(s-\mu)}{(1 / 2+s) \Gamma(1-s) \Gamma(1-s-\mu)} x^{-s-1} d s .
\end{aligned}
$$

Therefore, moving the contour of two latter integrals to the right via Cauchy's theorem, multiplying by $\sqrt{x}$, differentiating again and using (1.16), we obtain 


$$
\begin{gathered}
\frac{d}{d x}\left[\sqrt { x } \left[\left(x \frac{d}{d x}\right)^{2} \Phi+\left(1+\tau^{2}\right) \Phi+2 x \frac{d \Phi}{d x}\right.\right. \\
\left.\left.+\frac{1}{x}\left(x \frac{d}{d x}\right)^{2} \Phi-\frac{\mu^{2}}{x} \Phi\right]\right]=-\frac{x^{-3 / 2}}{2}\left[\left(x \frac{d}{d x}\right)^{2} \Phi-\mu^{2} \Phi\right] .
\end{gathered}
$$

Hence we arrive at the following operator equation

$2\left(x \frac{d}{d x}\right)^{3} \Phi+5\left(x \frac{d}{d x}\right)^{2} \Phi+2\left(2+\tau^{2}\right)\left(x \frac{d}{d x}\right) \Phi+2 \frac{d}{d x}\left(\left(x \frac{d}{d x}\right)^{2}-\mu^{2}\right) \Phi+\left(1+\tau^{2}\right) \Phi=0$.

Thus fulfilling the differentiation, we end up with (1.15). Lemma 2 is proved.

\section{BOUNDEDNESS AND INVERSION PROPERTIES FOR THE INDEX TRANSFORM (1.1)}

In this section we will investigate the mapping properties of the index transform (1.1). In fact, denoting by $C_{b}\left(\mathbb{R}_{+}\right)$the space of bounded continuous functions, we establish

Theorem 1. Let $\operatorname{Re} \mu<1 / 2$. The index transform (1.1) is welldefined as a bounded operator $F: L_{1-\nu, 1}\left(\mathbb{R}_{+}\right) \rightarrow C_{b}\left(\mathbb{R}_{+}\right), \nu \in(\operatorname{Re} \mu, 1 / 2)$ and the following norm inequality takes place

$$
\|F f\|_{C_{b}\left(\mathbb{R}_{+}\right)} \equiv \sup _{\tau \in \mathbb{R}_{+}}|(F f)(\tau)| \leq C_{\mu, \nu}\|f\|_{1-\nu, 1}
$$

where

$C_{\mu, \nu}=\frac{2^{-2 \nu}}{\pi \sqrt{\pi}} B(1-\nu, 1-\nu) \int_{\nu-i \infty}^{\nu+i \infty}\left|\frac{\Gamma(3 / 2-s) \Gamma(1 / 2-s) \Gamma(s-\mu)}{\Gamma(1-s-\mu)} d s\right|$

and $B(a, b)$ is the Euler beta-function [2], Vol. 1. Moreover, $(F f)(\tau) \rightarrow$ $0, \tau \rightarrow \infty$. Besides, if, in addition, $f \in L_{1-\nu, p}\left(\mathbb{R}_{+}\right), \varphi(x) \in L_{1-\nu, p}\left(\mathbb{R}_{+}\right), 1<$ $p \leq 2, \nu \in(\max (0, \operatorname{Re} \mu), 1 /(2 q)), \operatorname{Re} \mu<1 /(2 q), q=p /(p-1)$, where

$$
\varphi(x)=\frac{1}{2 \pi i} \int_{\nu-i \infty}^{\nu+i \infty} \frac{\Gamma(s-\mu) \Gamma(s) \Gamma(1 / 2-s)}{\Gamma(s-1 / 2) \Gamma(1-s) \Gamma(1-s-\mu)} f^{*}(1-s) x^{-s} d s,
$$


then

$$
(F f)(\tau)=\frac{\sqrt{\pi}}{\cosh (\pi \tau)} \int_{0}^{\infty} K_{i \tau}(\sqrt{x})\left[I_{i \tau}(\sqrt{x})+I_{-i \tau}(\sqrt{x})\right] \varphi(x) d x
$$

and integrals (2.3), (2.4) converge absolutely.

Proof. Indeed, recalling (1.4), (1.9), (1.12), using the duplication formula for the gamma-function and calculating an elementary integral with the hyperbolic function, we derive

$$
\begin{gathered}
|(F f)(\tau)| \leq \int_{0}^{\infty}|\Phi(x, \tau)||f(x)| d x \\
\leq \frac{1}{\pi \sqrt{\pi}} \int_{\nu-i \infty}^{\nu+i \infty}\left|\frac{\Gamma(3 / 2-s) \Gamma(1 / 2-s) \Gamma(s-\mu)}{\Gamma(1-s-\mu)} d s\right| \\
\times \int_{0}^{\infty}|f(x)| x^{-\nu} d x \int_{0}^{\infty} \frac{d y}{\cosh ^{2(1-\nu)}(y)} \\
=\frac{2^{-2 \nu}}{\pi \sqrt{\pi}} B(1-\nu, 1-\nu) \int_{\nu-i \infty}^{\nu+i \infty}\left|\frac{\Gamma(3 / 2-s) \Gamma(1 / 2-s) \Gamma(s-\mu)}{\Gamma(1-s-\mu)} d s\right|\|f\|_{1-\nu, 1} .
\end{gathered}
$$

The latter estimate proves (2.1). Furthermore, Fubini's theorem and the definition of the Mellin transform (1.6) yield the integral representation of $(F f)(\tau)$ in terms of the Fourier cosine transform of integrable function, namely,

$$
\begin{aligned}
(F f)(\tau)=\frac{1}{\pi i \sqrt{\pi}} \int_{0}^{\infty} & \frac{\cos (2 \tau y)}{\cosh ^{2}(y)} \int_{\nu-i \infty}^{\nu+i \infty} \frac{\Gamma(3 / 2-s) \Gamma(1 / 2-s) \Gamma(s-\mu)}{\Gamma(1-s-\mu)} \\
& \times f^{*}(1-s) \cosh ^{2 s}(y) d s d y .
\end{aligned}
$$

Hence it tends to zero, when $\tau \rightarrow \infty$ via the Riemann-Lebesgue lemma. Moreover, the Parseval equality (1.7) and (1.4) give the representation

$$
\begin{gathered}
(F f)(\tau)=\frac{1}{2 \pi i} \int_{\nu-i \infty}^{\nu+i \infty} \frac{\Gamma(1-s+i \tau) \Gamma(1-s-i \tau) \Gamma(1 / 2-s) \Gamma(s-\mu)}{\Gamma(1-s) \Gamma(1-s-\mu)} \\
\times f^{*}(1-s) d s .
\end{gathered}
$$

Hence, employing the Mellin-Barnes representation for the product of modified Bessel functions of the third kind (cf. relation (8.4.23.23) in [4], Vol. III) 


$$
\begin{gathered}
\frac{\sqrt{\pi}}{\cosh (\pi \tau)} K_{i \tau}(\sqrt{x})\left[I_{i \tau}(\sqrt{x})+I_{-i \tau}(\sqrt{x})\right] \\
=\frac{1}{2 \pi i} \int_{\nu-i \infty}^{\nu+i \infty} \frac{\Gamma(1 / 2-s)}{\Gamma(1-s)} \Gamma(s+i \tau) \Gamma(s-i \tau) x^{-s} d s, x>0
\end{gathered}
$$

and using again the Parseval equality (1.7), we find that (2.5) becomes the Lebedev index transform with the product of the modified Bessel functions [7] given by formula (2.4), where $\varphi(x)$ is defined by integral (2.3). Its absolute convergence for each $x>0$ can be verified via Hölder's inequality

$$
\begin{gathered}
\int_{\nu-i \infty}^{\nu+i \infty}\left|\frac{\Gamma(s-\mu) \Gamma(s) \Gamma(1 / 2-s)}{\Gamma(s-1 / 2) \Gamma(1-s) \Gamma(1-s-\mu)} f^{*}(1-s) x^{-s} d s\right| \\
\leq x^{-\nu}\left(\int_{\nu-i \infty}^{\nu+i \infty}\left|f^{*}(1-s)\right|^{q}|d s|\right)^{1 / q} \\
\times\left(\int_{\nu-i \infty}^{\nu+i \infty}\left|\frac{\Gamma(s-\mu) \Gamma(s) \Gamma(1 / 2-s)}{\Gamma(s-1 / 2) \Gamma(1-s) \Gamma(1-s-\mu)}\right|^{p}|d s|\right)^{1 / p}<\infty, q=\frac{p}{p-1} .
\end{gathered}
$$

The convergence of the latter integral by $s$ is justified, recalling the Stirling formula for the asymptotic behavior of the gamma-function, which gives

$$
\frac{\Gamma(s-\mu) \Gamma(s) \Gamma(1 / 2-s)}{\Gamma(s-1 / 2) \Gamma(1-s) \Gamma(1-s-\mu)}=O\left(|s|^{2 \nu-1}\right),|s| \rightarrow \infty,
$$

and $\nu$ is chosen from the interval $(\max (0, \operatorname{Re} \mu), 1 /(2 q)), \operatorname{Re} \mu<1 /(2 q)$. In order to establish the absolute convergence of the integral (2.4), we use the assumption $\varphi(x) \in L_{1-\nu, p}\left(\mathbb{R}_{+}\right)$and invoke the asymptotic formulae for the modified Bessel functions [2], Vol. 2 for fixed $\tau \in \mathbb{R}$, namely,

$$
\begin{gathered}
K_{i \tau}(\sqrt{x})\left[I_{i \tau}(\sqrt{x})+I_{-i \tau}(\sqrt{x})\right]=O(\log x), x \rightarrow 0+, \\
K_{i \tau}(\sqrt{x})\left[I_{i \tau}(\sqrt{x})+I_{-i \tau}(\sqrt{x})\right]=O\left(x^{-1 / 2}\right), x \rightarrow \infty .
\end{gathered}
$$

Then accordingly,

$$
\int_{0}^{\infty}\left|K_{i \tau}(\sqrt{x})\left[I_{i \tau}(\sqrt{x})+I_{-i \tau}(\sqrt{x})\right] \varphi(x)\right| d x
$$




$$
\begin{aligned}
& \leq\|\varphi\|_{1-\nu, p}\left(\int_{0}^{\infty}\left|K_{i \tau}(\sqrt{x})\left[I_{i \tau}(\sqrt{x})+I_{-i \tau}(\sqrt{x})\right]\right|^{q} x^{\nu q-1} d x\right)^{1 / q} \\
= & \|\varphi\|_{1-\nu, p}\left(\int_{0}^{1} O\left(x^{\nu q-1} \log ^{q} x\right) d x+\int_{1}^{\infty} O\left(x^{q(\nu-1 / 2)-1}\right) d x\right)^{1 / q}<\infty .
\end{aligned}
$$

Theorem 1 is proved.

Writing (2.4) in the form

$$
(F f)(\tau)=\frac{2 \sqrt{\pi}}{\cosh (\pi \tau)} \int_{0}^{\infty} K_{i \tau}(x)\left[I_{i \tau}(x)+I_{-i \tau}(x)\right] \varphi\left(x^{2}\right) x d x,
$$

we appeal to the Lebedev expansion theorem in [7], which implies the following representation of the antiderivative

$$
\int_{x}^{\infty} \varphi\left(y^{2}\right) y d y=\frac{1}{\pi^{2} \sqrt{\pi}} \int_{0}^{\infty} \tau \sinh (2 \pi \tau) K_{i \tau}^{2}(x)(F f)(\tau) d \tau, x>0
$$

which holds under condition $x \varphi\left(x^{2}\right) \in L_{1}\left((0,1) ; x^{-1 / 2} d x\right) \cap L_{1}\left((1, \infty) ; x^{1 / 2} d x\right)$. By straightforward substitutions we see that this condition is equivalent to (cf. (1.9)) $\varphi \in L_{5 / 4,1}(1, \infty) \cap L_{3 / 4,1}(0,1)$. Hence $(2.7)$ yields the equality

$$
\int_{x}^{\infty} \varphi(y) d y=\frac{2}{\pi^{2} \sqrt{\pi}} \int_{0}^{\infty} \tau \sinh (2 \pi \tau) K_{i \tau}^{2}(\sqrt{x})(F f)(\tau) d \tau .
$$

Then, appealing to relation (8.4.23.27) in [4, Vol. III and differentiating both sides of (2.8) with respect to $x$, we find

$$
\begin{aligned}
& \varphi(x)=-\frac{1}{2 \pi^{3} i} \frac{d}{d x} \int_{0}^{\infty} \tau \sinh (2 \pi \tau)(F f)(\tau) \\
& \times \int_{\nu-i \infty}^{\nu+i \infty} \frac{\Gamma(s) \Gamma(s+i \tau) \Gamma(s-i \tau)}{\Gamma(1 / 2+s)} x^{-s} d s d \tau,
\end{aligned}
$$

where $\nu>0$. Our goal now is to motivate the differentiation under integral sign in the right-hand side of (2.9). To do this, we first appeal to the Parseval equality (1.7) and relation (8.4.23.3) in [4], Vol. III in order to rewrite the integral with respect to $s$ in (2.10) as follows 
$\frac{1}{2 \pi i} \int_{\nu-i \infty}^{\nu+i \infty} \frac{\Gamma(s) \Gamma(s+i \tau) \Gamma(s-i \tau)}{\Gamma(1 / 2+s)} x^{-s} d s=\frac{1}{\sqrt{\pi}} \int_{0}^{\infty} e^{-y / 2-x / y} K_{i \tau}\left(\frac{y}{2}\right) \frac{d y}{y}$.

Hence after substitution of the right-hand side of the latter equality into (2.9) a formal differentiation means

$$
\varphi(x)=\frac{1}{\pi^{2} \sqrt{\pi}} \int_{0}^{\infty} \tau \sinh (2 \pi \tau)(F f)(\tau) \int_{0}^{\infty} e^{-y / 2-x / y} K_{i \tau}\left(\frac{y}{2}\right) \frac{d y d \tau}{y^{2}}
$$

It is allowed via the absolute and uniform convergence by $x \geq x_{0}>0$ of the iterated integral (2.10), which can be verified under the integrability condition $F f \in L_{1}\left(\mathbb{R}_{+} ; \tau e^{3 \pi \tau / 2} d \tau\right)$ and the Lebedev inequality for the modified Bessel function [5], p. 99

$$
\left|K_{i \tau}(y)\right| \leq \frac{y^{-1 / 4}}{\sqrt{\sinh (\pi \tau)}}, y, \tau>0 .
$$

Indeed, with the use of relation (2.3.16.1) in 4, Vol. I we have

$$
\begin{gathered}
\int_{0}^{\infty} \tau \sinh (2 \pi \tau)|(F f)(\tau)| \int_{0}^{\infty} e^{-y / 2-x / y}\left|K_{i \tau}\left(\frac{y}{2}\right)\right| \frac{d y d \tau}{y^{2}} \\
\leq \int_{0}^{\infty} \tau e^{3 \pi \tau / 2}|(F f)(\tau)| \int_{0}^{\infty} e^{-y-x_{0} /(2 y)} \frac{d y d \tau}{y^{9 / 4}} \\
=2^{13 / 8} x_{0}^{-5 / 8} K_{5 / 4}\left(\sqrt{2 x_{0}}\right) \int_{0}^{\infty} \tau e^{3 \pi \tau / 2}|(F f)(\tau)| d \tau<\infty .
\end{gathered}
$$

Moreover, one can change the order of integration in (2.10) due to Fubini's theorem to get

$$
\varphi(x)=\frac{1}{\pi^{2} \sqrt{\pi}} \int_{0}^{\infty} e^{-y / 2-x / y} \int_{0}^{\infty} \tau \sinh (2 \pi \tau) K_{i \tau}\left(\frac{y}{2}\right)(F f)(\tau) \frac{d \tau d y}{y^{2}}
$$

This equality guarantees the condition $\varphi \in L_{\nu+1,1}\left(\mathbb{R}_{+}\right), \nu>1 / 4$. In fact, we have with (2.11)

$$
\begin{gathered}
\|\varphi\|_{1+\nu, 1}=\int_{0}^{\infty}|\varphi(x)| x^{\nu} d x \leq \frac{\Gamma(\nu+3 / 4) \Gamma(\nu-1 / 4) 2^{\nu+3 / 4}}{\pi^{2} \sqrt{\pi}} \\
\times \int_{0}^{\infty} \tau \sqrt{\sinh (\pi \tau)} \cosh (\pi \tau)|(F f)(\tau)| d \tau<\infty .
\end{gathered}
$$

Therefore, recalling the Parseval equality (1.7), equality (2.12) can be written in the form 


$$
\begin{gathered}
x \varphi(x)=\frac{1}{2 \pi^{3} i} \int_{\nu-i \infty}^{\nu+i \infty} \frac{\Gamma(1+s)}{\Gamma(1 / 2+s)} x^{-s} \int_{0}^{\infty} \tau \sinh (2 \pi \tau)(F f)(\tau) \\
\times \Gamma(s+i \tau) \Gamma(s-i \tau) d \tau d s .
\end{gathered}
$$

Besides, applying the Mellin transform to both sides (2.13) (cf. (2.3)), we obtain after a slight simplification

$$
\begin{gathered}
\frac{\Gamma(s+1-\mu) \Gamma(-1 / 2-s)}{\Gamma(-s) \Gamma(-s-\mu)} f^{*}(-s)=\frac{1}{\pi^{2}} \int_{0}^{\infty} \tau \sinh (2 \pi \tau)(F f)(\tau) \\
\times \Gamma(s+i \tau) \Gamma(s-i \tau) d \tau
\end{gathered}
$$

Hence, employing the reduction formula for the gamma-function and the inverse Mellin transform (1.8) under the integrability condition $s f^{*}(-s) \in L_{1}(\nu-i \infty, \nu+i \infty)$, we deduce from (2.14)

$$
\begin{gathered}
-\frac{1}{2 \pi i} \int_{\nu-i \infty}^{\nu+i \infty} s f^{*}(-s) x^{-s} d s=\frac{1}{2 \pi^{3} i} \int_{\nu-i \infty}^{\nu+i \infty} \frac{\Gamma(1-s) \Gamma(-s-\mu)}{\Gamma(s+1-\mu) \Gamma(-1 / 2-s)} x^{-s} \\
\quad \times \int_{0}^{\infty} \tau \sinh (2 \pi \tau)(F f)(\tau) \Gamma(s+i \tau) \Gamma(s-i \tau) d \tau d s
\end{gathered}
$$

The left-hand side of (2.15) can be treated with the use of the operational properties for the Mellin transform under corresponding conditions, and in the right-hand side we interchange the order of integration, justifying this passage below. Consequently, we obtain for almost all $x>0$ the equality

$$
x \frac{d}{d x}\left[f\left(\frac{1}{x}\right)\right]=\frac{1}{\pi^{2}} \int_{0}^{\infty} \tau \sinh (2 \pi \tau) S(x, \tau)(F f)(\tau) d \tau,
$$

where the kernel $S(x, \tau)$ is defined in terms of the Mellin-Barnes integral

$$
S(x, \tau)=\frac{1}{2 \pi i} \int_{\nu-i \infty}^{\nu+i \infty} \frac{\Gamma(s+i \tau) \Gamma(s-i \tau) \Gamma(1-s) \Gamma(-s-\mu)}{\Gamma(s+1-\mu) \Gamma(-1 / 2-s)} x^{-s} d s,
$$

and the vertical line $(\nu-i \infty, \nu+i \infty)$ in the complex plane $s$ separates left- and right-hand side simple poles of the gamma-functions under the assumption $0<\nu<\min (-\operatorname{Re} \mu, 1), \mu \notin \mathbb{Z}$.

In the meantime, to justify the interchange of the order of integration in (2.15), we appeal again to the Parseval equality (1.7) and relation 
(8.4.23.3) in 4], Vol. III to write the right-hand side of (2.15) in the form

$$
\begin{gathered}
\frac{1}{2 \pi^{3} i} \int_{\nu-i \infty}^{\nu+i \infty} \frac{\Gamma(1-s) \Gamma(-s-\mu)}{\Gamma(s+1-\mu) \Gamma(-1 / 2-s)} x^{-s} \\
\times \int_{0}^{\infty} \tau \sinh (2 \pi \tau)(F f)(\tau) \Gamma(s+i \tau) \Gamma(s-i \tau) d \tau d s \\
=\frac{1}{\pi^{5 / 2}} \int_{0}^{\infty} e^{-y / 2} h\left(\frac{x}{y}\right) \int_{0}^{\infty} \tau \sinh (2 \pi \tau) K_{i \tau}\left(\frac{y}{2}\right)(F f)(\tau) \frac{d \tau d y}{y}
\end{gathered}
$$

where

$$
h(x)=\frac{1}{2 \pi i} \int_{\nu-i \infty}^{\nu+i \infty} \frac{\Gamma(s+1 / 2) \Gamma(1-s) \Gamma(-s-\mu)}{\Gamma(s+1-\mu) \Gamma(-1 / 2-s)} x^{-s} d s
$$

and $1 / 4<\nu<\min (-\operatorname{Re} \mu, 1)$. But for $\mu \notin \mathbb{Z}$ the integral (2.19) can be calculated in terms of the generalized hypergeometric functions by means of the Slater theorem [4], Vol. III. Precisely, we find the value

$$
\begin{gathered}
h(x)=\frac{3 \Gamma(-1-\mu)}{8 x \Gamma(2-\mu)}{ }_{2} F_{2}\left(\frac{3}{2}, \frac{5}{2} ; 2+\mu, 2-\mu ;-\frac{1}{x}\right) \\
+\left(\frac{x}{4}\right)^{\mu} \frac{\sqrt{\pi} \Gamma(1+\mu)}{\Gamma(1-\mu) \Gamma(\mu-1 / 2)}{ }_{2} F_{2}\left(\frac{1}{2}-\mu, \frac{3}{2}-\mu ;-\mu, 1-2 \mu ;-\frac{1}{x}\right) .
\end{gathered}
$$

Meanwhile, the interchange of the order of integration in the righthand side of (2.18) by Fubini's theorem is motivated by the following estimate (see (2.11), (2.19))

$$
\begin{gathered}
\int_{0}^{\infty} e^{-y / 2}\left|h\left(\frac{x}{y}\right)\right| \int_{0}^{\infty} \tau \sinh (2 \pi \tau)\left|K_{i \tau}\left(\frac{y}{2}\right)(F f)(\tau)\right| \frac{d \tau d y}{y} \\
\leq \frac{x^{-\nu} 2^{1 / 4}}{\pi} \int_{0}^{\infty} e^{-y / 2} y^{\nu-5 / 4} d y \int_{\nu-i \infty}^{\nu+i \infty}\left|\frac{\Gamma(s+1 / 2) \Gamma(1-s) \Gamma(-s-\mu)}{\Gamma(s+1-\mu) \Gamma(-1 / 2-s)} d s\right| \\
\quad \times \int_{0}^{\infty} \tau \sqrt{\sinh (\pi \tau)} \cosh (\pi \tau)|(F f)(\tau)| d \tau<\infty .
\end{gathered}
$$

Therefore we end up with the equality (2.16), where the kernel (2.17) can be written in the form 


$$
S(x, \tau)=\frac{1}{\sqrt{\pi}} \int_{0}^{\infty} e^{-y / 2} h\left(\frac{x}{y}\right) K_{i \tau}\left(\frac{y}{2}\right) \frac{d y}{y} .
$$

Moreover, it can be calculated explicitly, substituting (2.20) in the right-hand side of (2.21) and employing relation (3.35.9.3) in [8]. In fact, we get

$$
\begin{gathered}
S(x, \tau)=\frac{3 \sqrt{\pi} \tau \Gamma(-1-\mu)}{4 x \Gamma(2-\mu) \sinh (\pi \tau)}{ }_{3} F_{2}\left(1+i \tau, 1-i \tau, \frac{5}{2} ; 2+\mu, 2-\mu ;-\frac{1}{x}\right) \\
+\left(\frac{x}{4}\right)^{\mu} \frac{\sqrt{\pi} \Gamma(1+\mu) \Gamma(-\mu-i \tau) \Gamma(-\mu+i \tau)}{\Gamma(1-\mu) \Gamma(\mu-1 / 2) \Gamma(1 / 2-\mu)} \\
\times{ }_{3} F_{2}\left(-\mu-i \tau,-\mu+i \tau, \frac{3}{2}-\mu ;-\mu, 1-2 \mu ;-\frac{1}{x}\right) .
\end{gathered}
$$

Finally, returning to (2.16) and making a simple substitution, we come up with the following inversion formula for the index transform (1.1)

$$
f(x)=-\frac{1}{\pi^{2}} \int_{1 / x}^{\infty} \int_{0}^{\infty} \tau \sinh (2 \pi \tau) S(y, \tau)(F f)(\tau) \frac{d \tau d y}{y} .
$$

One can change the order of integration in the right-hand side of (2.23) due to the estimate (see (2.19), (2.21))

$$
\begin{gathered}
\int_{1 / x}^{\infty} \int_{0}^{\infty} \tau \sinh (2 \pi \tau)|S(y, \tau)(F f)(\tau)| \frac{d \tau d y}{y} \\
\leq C \|\left. F f\right|_{L_{1}\left(\mathbb{R}_{+} ; \tau e^{3 \pi \tau / 2} d \tau\right)} \int_{0}^{\infty} e^{-u} u^{\nu-5 / 4} d u \int_{1 / x}^{\infty} y^{-\nu-1} d y<\infty, \nu>\frac{1}{4},
\end{gathered}
$$

where $C>0$ is an absolute constant. Hence from (2.22) and relation (1.16.1.1) in [4], Vol. III

$$
\begin{aligned}
& \int_{1 / x}^{\infty} S(y, \tau) \frac{d y}{y}=\frac{3 x \sqrt{\pi} \tau \Gamma(-1-\mu)}{4 \Gamma(2-\mu) \sinh (\pi \tau)}{ }_{4} F_{3}\left(1+i \tau, 1-i \tau, \frac{5}{2}, 1 ; 2+\mu, 2-\mu, 2 ;-x\right) \\
&-\frac{1}{(4 x)^{\mu}} \frac{\sqrt{\pi} \Gamma(\mu) \Gamma(-\mu-i \tau) \Gamma(-\mu+i \tau)}{\Gamma(1-\mu) \Gamma(\mu-1 / 2) \Gamma(1 / 2-\mu)} \\
& \quad \times{ }_{3} F_{2}\left(-\mu-i \tau,-\mu+i \tau, \frac{3}{2}-\mu ; 1-\mu, 1-2 \mu ;-x\right),
\end{aligned}
$$

and after a simple substitution formula (2.23) can be written as follows 


$$
\begin{gathered}
f(x)=\frac{1}{\pi \sqrt{\pi}} \int_{0}^{\infty}\left[\frac{\Gamma(\mu) \Gamma(-\mu-i \tau) \Gamma(-\mu+i \tau) \sinh (2 \pi \tau)}{(4 x)^{\mu} \Gamma(1-\mu) \Gamma(\mu-1 / 2) \Gamma(1 / 2-\mu)}\right. \\
\times{ }_{3} F_{2}\left(-\mu-i \tau,-\mu+i \tau, \frac{3}{2}-\mu ; 1-\mu, 1-2 \mu ;-x\right) \\
\left.-\frac{3 x \tau \cosh (\pi \tau) \Gamma(-1-\mu)}{2 \Gamma(2-\mu)}{ }_{4} F_{3}\left(1+i \tau, 1-i \tau, \frac{5}{2}, 1 ; 2+\mu, 2-\mu, 2 ;-x\right)\right] \\
\times(F f)(\tau) \tau d \tau .
\end{gathered}
$$

The hypergeometric functions in (2.25) can be expressed in terms of the associated Legendre functions. To do this, we, firstly, appeal to the differential property for the generalized hypergeometric function [2], Vol. I to have the formula

$$
\begin{gathered}
{ }_{3} F_{2}\left(-\mu-i \tau,-\mu+i \tau, \frac{3}{2}-\mu ; 1-\mu, 1-2 \mu ;-x\right)=\frac{x^{\mu+1 / 2}}{1 / 2-\mu} \\
\times \frac{d}{d x}\left[x_{3}^{1 / 2-\mu} F_{2}\left(-\mu-i \tau,-\mu+i \tau, \frac{1}{2}-\mu ; 1-\mu, 1-2 \mu ;-x\right)\right] .
\end{gathered}
$$

However, the generalized hypergeometric function on the right-hand side of the latter equality can be written in terms of the squares of the associated Legendre functions (cf. 44, Vol. III, Entries 7.4.1.26 and 7.3.1.40 ), taking into account the fundamental identity [2], Vol. I

$$
P_{\nu}^{\mu}(z)=P_{-\nu-1}^{\mu}(z)
$$

Hence we obtain

$$
\begin{aligned}
& { }_{3} F_{2}\left(-\mu-i \tau,-\mu+i \tau, \frac{1}{2}-\mu ; 1-\mu, 1-2 \mu ;-x\right)=\left(\frac{x}{4}\right)^{\mu} \frac{\Gamma^{2}(1-\mu)}{2 i \tau} \\
& \times\left[(i \tau+\mu)\left[P_{-i \tau}^{\mu}(\sqrt{1+x})\right]^{2}+(i \tau-\mu)\left[P_{i \tau}^{\mu}(\sqrt{1+x})\right]^{2}\right] \cdot(2.27)
\end{aligned}
$$

In the same manner we write

$$
{ }_{4} F_{3}\left(1+i \tau, 1-i \tau, \frac{5}{2}, 1 ; 2+\mu, 2-\mu, 2 ;-x\right)
$$




$$
=\frac{2}{3 \sqrt{x}} \frac{d}{d x}\left[x^{3 / 2}{ }_{4} F_{3}\left(1+i \tau, 1-i \tau, \frac{3}{2}, 1 ; 2+\mu, 2-\mu, 2 ;-x\right)\right] .
$$

Moreover, in turn, (see [4], Vol. III, Entry 1.16.1.1)

$$
\begin{gathered}
{ }_{4} F_{3}\left(1+i \tau, 1-i \tau, \frac{3}{2}, 1 ; 2+\mu, 2-\mu, 2 ;-x\right) \\
=(\mu-1) x^{\mu-1} \int_{x}^{\infty} y^{-\mu}{ }_{4} F_{3}\left(1+i \tau, 1-i \tau, \frac{3}{2}, 1 ; 2+\mu, 1-\mu, 2 ;-y\right) d y .
\end{gathered}
$$

But the formula in [4, Vol. III, Entry 7.5.1.5 says

$$
\begin{gathered}
{ }_{4} F_{3}\left(1+i \tau, 1-i \tau, \frac{3}{2}, 1 ; 2+\mu, 1-\mu, 2 ;-y\right) \\
={ }_{2} F_{1}\left(1+i \tau, 1-i \tau ; 2+\mu ; \frac{1-\sqrt{1+y}}{2}\right){ }_{2} F_{1}\left(1+i \tau, 1-i \tau ; 1-\mu ; \frac{1-\sqrt{1+y}}{2}\right) .
\end{gathered}
$$

Besides, via the formula in [4], Vol. III, Entry 7.3.1.92 we find the equalities

$$
\begin{gathered}
{ }_{2} F_{1}\left(1+i \tau, 1-i \tau ; 1-\mu ; \frac{1-\sqrt{1+y}}{2}\right) \\
=\frac{\Gamma(1-\mu)}{i \tau}\left(\frac{\sqrt{y}}{\sqrt{1+y}+1}\right)^{\mu}\left[(i \tau-\mu) P_{-1-i \tau}^{\mu}(\sqrt{1+y})-\frac{\sqrt{y}}{\sqrt{1+y}+1} P_{-1-i \tau}^{1+\mu}(\sqrt{1+y})\right] \\
{ }_{2} F_{1}\left(1+i \tau, 1-i \tau ; 2+\mu ; \frac{1-\sqrt{1+y}}{2}\right) \\
=\frac{\Gamma(2+\mu)}{i \tau}\left(\frac{\sqrt{y}}{\sqrt{1+y}+1}\right)^{-(1+\mu)}\left[(1+i \tau+\mu) P_{-1-i \tau}^{-1-\mu}(\sqrt{1+y})\right. \\
\left.-\frac{\sqrt{y}}{\sqrt{1+y}+1} P_{-1-i \tau}^{-\mu}(\sqrt{1+y})\right] .
\end{gathered}
$$

Finally, minding (2.26), we get

$$
\begin{aligned}
& { }_{4} F_{3}\left(1+i \tau, 1-i \tau, \frac{3}{2}, 1 ; 2+\mu, 1-\mu, 2 ;-y\right)=-\frac{\Gamma(2+\mu) \Gamma(1-\mu)}{\tau^{2} \sqrt{y}(\sqrt{1+y}+1)} \\
& \quad \times\left[(1+i \tau+\mu)(\sqrt{1+y}+1) P_{i \tau}^{-1-\mu}(\sqrt{1+y})-\sqrt{y} P_{i \tau}^{-\mu}(\sqrt{1+y})\right]
\end{aligned}
$$




$$
\times\left[(i \tau-\mu)(\sqrt{1+y}+1) P_{i \tau}^{\mu}(\sqrt{1+y})-\sqrt{y} P_{i \tau}^{1+\mu}(\sqrt{1+y})\right] .
$$

We summarize these results as the following inversion theorem.

Theorem 2. Let $\operatorname{Re} \mu<0, \mu \notin \mathbb{Z}, \min (-\operatorname{Re} \mu, 1)>1 / 4$, the conditions of Theorem 1 hold and $\varphi \in L_{3 / 4,1}(0,1) \cap L_{5 / 4,1}(1, \infty), f \in$ $L_{-\nu, 1}\left(\mathbb{R}_{+}\right), \min (-\operatorname{Re} \mu, 1)>\nu>1 / 4, F f \in L_{1}\left(\mathbb{R}_{+} ; \tau e^{3 \pi \tau / 2} d \tau\right)$. Let, besides, the Mellin transform $f^{*}(-\nu-i t)$ be such that $(\nu+i t) f^{*}(-\nu-$ it $) \in L_{1}(\mathbb{R})$. Then for all $x>0$ the inversion formula $(2.25)$ holds.

\section{INDEX TRANSFORM $(1.2)$}

In this section we investigate the boundedness properties of the operator (1.2) and establish the inversion formula for this transformation. We begin with

Theorem 3. Let $\operatorname{Re} \mu<1 / 2$. The index transform (1.2) is welldefined as a bounded operator $G: L_{1}\left(\mathbb{R}_{+}\right) \rightarrow L_{\nu, \infty}\left(\mathbb{R}_{+}\right), \nu \in(\operatorname{Re} \mu, 1 / 2)$ and the following norm inequality takes place

$$
\|G g\|_{\nu, \infty} \equiv \text { ess } \sup _{x>0}\left|x^{\nu}(G g)(x)\right| \leq C_{\mu, \nu}\|g\|_{L_{1}\left(\mathbb{R}_{+}\right)},
$$

where $C_{\mu, \nu}$ is defined by (2.2). Moreover, if, besides, $G g \in L_{1-\gamma, 1}\left(\mathbb{R}_{+}\right), 1 / 2<$ $\gamma<1-\operatorname{Re} \mu, G^{*} g$ is analytic in the strip $\operatorname{Re} \mu<\operatorname{Re} s<1-\operatorname{Re} \mu$ and

$\sup _{\mu_{0} \leq \operatorname{Re} \leq \leq \mu_{1}}\left|\left(G^{*} g\right)(1-\operatorname{Re} s+i t)\right| \rightarrow 0, \quad|t| \rightarrow \infty,\left[\mu_{0}, \mu_{1}\right] \subset(\operatorname{Re} \mu, 1-\operatorname{Re} \mu)$,

where $\max (\operatorname{Re} \mu, 0)<\mu_{0}<1 / 2<\mu_{1}$, then for all $y>0$

$$
\begin{aligned}
& \frac{1}{2 \pi i} \int_{\nu-i \infty}^{\nu+i \infty} \frac{\Gamma(s) \Gamma(s-\mu) \Gamma(1 / 2-s)}{\Gamma(s-1 / 2) \Gamma(1-s-\mu)}\left(G^{*} g\right)(1-s) y^{-s} d s \\
= & \sqrt{\pi} e^{y / 2} \int_{0}^{\infty} K_{i \tau}\left(\frac{y}{2}\right) \frac{g(\tau)}{\cosh (\pi \tau)} d \tau, \quad \nu \in\left[\mu_{0}, 1 / 2\right) .
\end{aligned}
$$

Proof. Indeed, as we see from (1.4) the kernel $\Phi(x, \tau)$ has a bound

$$
|\Phi(x, \tau)| \leq C_{\mu, \nu} x^{-\nu}, x>0
$$

where $C_{\mu, \nu}$ is defined by $(2.2)$, we have

$$
|(G g)(x)| \leq C_{\mu, \nu} x^{-\nu} \int_{\mathbb{R}_{+}}|g(\tau)| d \tau=C_{\mu, \nu} x^{-\nu}|| g \|_{L_{1}\left(\mathbb{R}_{+}\right)},
$$

and (3.1) holds. Further, taking the Mellin transform (1.6) of both sides of (1.2) at the point $1-s$ under the condition $G g \in L_{1-\gamma, 1}\left(\mathbb{R}_{+}\right), 1 / 2<$ 
$\gamma<1-\operatorname{Re} \mu$, we change the order of integration on the right-hand side of the obtained equality by Fubini's theorem. Moreover, taking into account (1.4), we derive

$$
\frac{\Gamma(s) \Gamma(s-\mu)}{\Gamma(s-1 / 2) \Gamma(1-s-\mu)}\left(G^{*} g\right)(1-s)=\int_{0}^{\infty} \Gamma(s+i \tau) \Gamma(s-i \tau) g(\tau) d \tau .
$$

Meanwhile, employing relation (8.4.23.5) in 4, Vol. III

$\frac{\sqrt{\pi}}{\cosh (\pi \tau)} e^{x / 2} K_{i \tau}\left(\frac{x}{2}\right)=\frac{1}{2 \pi i} \int_{\nu-i \infty}^{\nu+i \infty} \Gamma(s+i \tau) \Gamma(s-i \tau) \Gamma(1 / 2-s) x^{-s} d s$

we multiply $(3.4)$ by $\Gamma(1 / 2-s)$ and then take the inverse Mellin transform (1.8) of both sides of the obtained equality over the vertical line $(\nu-i \infty, \nu+i \infty), \nu \in\left[\mu_{0}, 1 / 2\right)$. This is indeed possible via (3.2) and conditions of the theorem. Thus we establish (3.3), where the integral in the left-hand side converges absolutely. Theorem 3 is proved.

The inversion formula for the index transform (1.2) is given by

Theorem 4. Let $\operatorname{Re} \mu<1 / 2, \mu \notin \mathbb{Z}, g(z / i)$ be an even analytic function in the strip $D=\{z \in \mathbb{C}:|\operatorname{Re} z| \leq \alpha<1 / 2\}$, such that $g(0)=$ $g^{\prime}(0)=0$ and $g(z / i)$ be absolutely integrable over any vertical line in $D$. If $G g \in L_{1-\nu, 1}\left(\mathbb{R}_{+}\right), \nu \in\left[\mu_{0}, 1 / 2\right), \mu_{0} \in(\max (\operatorname{Re} \mu, 0), 1 / 2)$, then for all $x \in \mathbb{R}_{+}$the inversion formula holds for the index transform (1.2)

$$
\begin{gathered}
g(x)=\lim _{\varepsilon \rightarrow 0+} \frac{1}{\pi^{2}} x \sinh (2 \pi x) \int_{0}^{\infty}\left[\frac{\sqrt{\pi} \Gamma(\varepsilon-i x) \Gamma(\varepsilon+i x)}{2 \mu \Gamma(1 / 2+\varepsilon)}\right. \\
\times{ }_{4} F_{3}\left(\frac{1}{2}, \frac{3}{2}, \varepsilon-i x, \varepsilon+i x ; 1+\mu, 1-\mu, \frac{1}{2}+\varepsilon ;-u\right) \\
+\frac{\pi 4^{\mu} \Gamma(\mu) \Gamma(\varepsilon-\mu-i x) \Gamma(\varepsilon-\mu+i x)}{\Gamma(1 / 2+\varepsilon-\mu) \Gamma(\mu-1 / 2) \Gamma(1-\mu)} \\
\left.\times{ }_{4} F_{3}\left(\frac{1}{2}-\mu, \frac{3}{2}-\mu, \varepsilon-\mu-i x, \varepsilon-\mu+i x ; 1-\mu, 1-2 \mu, \frac{1}{2}+\varepsilon-\mu ;-u\right)\right] \\
\times(G f)(u) d u .
\end{gathered}
$$

Proof. Indeed, recalling (3.3), we write its left-hand side, appealing to the Parseval equality (1.7), to get 


$$
\begin{gathered}
\frac{1}{2 \pi i} \int_{\nu-i \infty}^{\nu+i \infty} \frac{\Gamma(s) \Gamma(s-\mu) \Gamma(1 / 2-s)}{\Gamma(s-1 / 2) \Gamma(1-s-\mu)}\left(G^{*} g\right)(1-s) y^{-s} d s \\
=\int_{0}^{\infty} U_{\mu}(y u)(G f)(u) d u,
\end{gathered}
$$

where

$$
U_{\mu}(y)=\frac{1}{2 \pi i} \int_{\nu-i \infty}^{\nu+i \infty} \frac{\Gamma(s) \Gamma(s-\mu) \Gamma(1 / 2-s)}{\Gamma(s-1 / 2) \Gamma(1-s-\mu)} y^{-s} d s, y>0 .
$$

The function $U_{\mu}(y), \mu \notin \mathbb{Z}$ can be calculated explicitly by Slater's theorem in terms of a combination of two hypergeometric functions ${ }_{2} F_{2}$. Precisely, we obtain

$$
\begin{gathered}
U_{\mu}(y)=\frac{1}{2 \mu}{ }_{2} F_{2}\left(\frac{1}{2}, \frac{3}{2} ; 1+\mu, 1-\mu ;-y\right) \\
+\left(\frac{y}{4}\right)^{-\mu} \frac{\sqrt{\pi} \Gamma(\mu)}{\Gamma(\mu-1 / 2) \Gamma(1-\mu)}{ }_{2} F_{2}\left(\frac{1}{2}-\mu, \frac{3}{2}-\mu ; 1-\mu, 1-2 \mu ;-y\right) .
\end{gathered}
$$

Returning to (3.3), we substitute its left-hand side by the right-hand side of (3.7) and multiply both sides of the obtained equality by $e^{-y / 2} K_{i x}(y / 2) y^{\varepsilon-1}$ for some positive $\varepsilon \in(0,1)$. Then, integrating with respect to $y$ over $(0, \infty)$, we appeal to the asymptotic behavior of the hypergeometric functions at infinity [2, Vol. I and the condition $G g \in L_{1-\nu, 1}\left(\mathbb{R}_{+}\right), \nu \in$ $\left[\mu_{0}, 1 / 2\right)$ to justify the interchange of the order of integration in the left-hand side due to the absolute convergence of the iterated integral. Finally, the inner integral is calculated with the use of formula 3.35.9.3 in [8], and we derive the equality

$$
\begin{gathered}
\int_{0}^{\infty} \int_{0}^{\infty} e^{-y / 2} K_{i x}(y / 2) y^{\varepsilon-1} U_{\mu}(y u)(G f)(u) d y d u \\
=\int_{0}^{\infty}\left[\frac{\sqrt{\pi} \Gamma(\varepsilon-i x) \Gamma(\varepsilon+i x)}{2 \mu \Gamma(1 / 2+\varepsilon)}{ }_{4} F_{3}\left(\frac{1}{2}, \frac{3}{2}, \varepsilon-i x, \varepsilon+i x ; 1+\mu, 1-\mu, \frac{1}{2}+\varepsilon ;-u\right)\right. \\
+\frac{\pi 4^{\mu} \Gamma(\mu) \Gamma(\varepsilon-\mu-i x) \Gamma(\varepsilon-\mu+i x)}{\Gamma(1 / 2+\varepsilon-\mu) \Gamma(\mu-1 / 2) \Gamma(1-\mu)} \\
\left.\times{ }_{4} F_{3}\left(\frac{1}{2}-\mu, \frac{3}{2}-\mu, \varepsilon-\mu-i x, \varepsilon-\mu+i x ; 1-\mu, 1-2 \mu, \frac{1}{2}+\varepsilon-\mu ;-u\right)\right]
\end{gathered}
$$




$$
\times(G f)(u) d u=\int_{0}^{\infty} K_{i x}\left(\frac{y}{2}\right) y^{\varepsilon-1} \int_{0}^{\infty} K_{i \tau}\left(\frac{y}{2}\right) \frac{g(\tau)}{\cosh (\pi \tau)} d \tau d y .
$$

In the meantime, the right-hand side of latter equality in (3.8) can be treated, using the evenness of $g$ and the representation of the modified Bessel function $K_{z}(y)$ in terms of the modified Bessel function of the first kind $I_{z}(y)$ [2], Vol. II. Hence with a simple substitution we find

$$
\begin{aligned}
& \frac{1}{2} \int_{0}^{\infty} K_{i x}\left(\frac{y}{2}\right) y^{\varepsilon-1} \int_{-\infty}^{\infty} K_{i \tau}\left(\frac{y}{2}\right) \frac{g(\tau)}{\cosh (\pi \tau)} d \tau d y \\
= & \pi i \int_{0}^{\infty} K_{i x}\left(\frac{y}{2}\right) y^{\varepsilon-1} \int_{-i \infty}^{i \infty} I_{z}\left(\frac{y}{2}\right) \frac{g(z / i)}{\sin (2 \pi z)} d z d y .
\end{aligned}
$$

On the other hand, according to our assumption $g(z / i)$ is analytic and integrable in the vertical strip $0 \leq \operatorname{Re} z \leq \alpha<1 / 2, g(0)=g^{\prime}(0)=0$. Hence, appealing to the inequality for the modified Bessel function of the first kind (see [5], p. 93)

$$
\left|I_{z}(y)\right| \leq I_{\operatorname{Re} z}(y) e^{\pi|\operatorname{Im} z| / 2}, 0<\operatorname{Re} z \leq \alpha,
$$

one can move the contour to the right in the latter integral in (3.8). Then

$$
\begin{aligned}
& \pi i \int_{0}^{\infty} K_{i x}\left(\frac{y}{2}\right) y^{\varepsilon-1} \int_{-i \infty}^{i \infty} I_{z}\left(\frac{y}{2}\right) \frac{g(z / i)}{\sin (2 \pi z)} d z d y \\
= & \pi i \int_{0}^{\infty} K_{i x}\left(\frac{y}{2}\right) y^{\varepsilon-1} \int_{\alpha-i \infty}^{\alpha+i \infty} I_{z}\left(\frac{y}{2}\right) \frac{g(z / i)}{\sin (2 \pi z)} d z d y .
\end{aligned}
$$

Now $\operatorname{Re} z>0$, and it is possible to pass to the limit under the integral sign when $\varepsilon \rightarrow 0$ and to change the order of integration due to the absolute and uniform convergence. Therefore the value of the integral (see relation $(2.16 .28 .3)$ in [4], Vol. II)

$$
\int_{0}^{\infty} K_{i x}(y) I_{z}(y) \frac{d y}{y}=\frac{1}{x^{2}+z^{2}}
$$

leads us to the equalities

$$
\begin{gathered}
\lim _{\varepsilon \rightarrow 0} \pi i \int_{0}^{\infty} K_{i x}\left(\frac{y}{2}\right) y^{\varepsilon-1} \int_{-i \infty}^{i \infty} I_{z}\left(\frac{y}{2}\right) \frac{g(z / i)}{\sin (2 \pi z)} d z d y \\
=\pi i \int_{\alpha-i \infty}^{\alpha+i \infty} \frac{g(z / i)}{\left(x^{2}+z^{2}\right) \sin (2 \pi z)} d z \\
=\frac{\pi i}{2}\left(\int_{-\alpha+i \infty}^{-\alpha-i \infty}+\int_{\alpha-i \infty}^{\alpha+i \infty}\right) \frac{g(z / i) d z}{(z-i x) z \sin (2 \pi z)} .
\end{gathered}
$$


Hence conditions of the theorem allow to apply the Cauchy formula in the right-hand side of the latter equality in (3.8). Thus

$$
\begin{gathered}
\lim _{\varepsilon \rightarrow 0} \pi i \int_{0}^{\infty} K_{i x}\left(\frac{y}{2}\right) y^{\varepsilon-1} \int_{-i \infty}^{i \infty} I_{z}\left(\frac{y}{2}\right) \frac{g(z / i)}{\sin (2 \pi z)} d z d y \\
=\frac{\pi^{2} g(x)}{x \sinh (2 \pi x)}, \quad x>0 .
\end{gathered}
$$

Therefore passing to the limit through equalities (3.8), we end up with the inversion formula (3.6), completing the proof of Theorem 4.

Remark 1. If the passage to the limit in (3.6) is permitted, the inversion formula takes the form

$$
\begin{gathered}
g(x)=\frac{1}{\pi \mu} \int_{0}^{\infty}\left[\cosh (\pi x)_{3} F_{2}\left(\frac{3}{2},-i x, i x ; 1+\mu, 1-\mu ;-u\right)\right. \\
+\frac{4^{\mu} \Gamma(1+\mu) \Gamma(-\mu-i x) \Gamma(-\mu+i x) x \sinh (2 \pi x)}{\Gamma(1 / 2-\mu) \Gamma(\mu-1 / 2) \Gamma(1-\mu)} \\
\left.\times{ }_{3} F_{2}\left(\frac{3}{2}-\mu,-\mu-i x,-\mu+i x ; 1-\mu, 1-2 \mu ;-u\right)\right](G f)(u) d u .
\end{gathered}
$$

\section{Boundary VALUe PROBLEM}

In this section the index transform (1.2) is employed to investigate the solvability of the boundary value problem for the following third order partial differential equation

$$
\begin{gathered}
2 x\left(y^{2}+x^{2}\left(1+\frac{1}{r}\right)\right) \frac{\partial^{3} u}{\partial x^{3}}+2 y\left(x^{2}+y^{2}\left(1+\frac{1}{r}\right)\right) \frac{\partial^{3} u}{\partial y^{3}} \\
+2 x\left(x^{2}+y^{2}\left(1+\frac{3}{r}\right)\right) \frac{\partial^{3} u}{\partial x \partial y^{2}}+2 y\left(y^{2}+x^{2}\left(1+\frac{3}{r}\right)\right) \frac{\partial^{3} u}{\partial y \partial x^{2}} \\
+\left(5 y^{2}+3 x^{2}\left(3+\frac{2}{r}\right)\right) \frac{\partial^{2} u}{\partial x^{2}}+\left(5 x^{2}+3 y^{2}\left(3+\frac{2}{r}\right)\right) \frac{\partial^{2} u}{\partial y^{2}} \\
+4 x y\left(2+\frac{3}{r}\right) \frac{\partial^{2} u}{\partial x \partial y}+2\left(4+\frac{1-\mu^{2}}{r}\right)\left(x \frac{\partial u}{\partial x}+y \frac{\partial u}{\partial y}\right)+u=0
\end{gathered}
$$

where $(x, y) \in \mathbb{R}^{2} \backslash\{0\}, r=\sqrt{x^{2}+y^{2}}$. Writing (4.1) in polar coordinates $(r, \theta)$, we end up with the equation 


$$
\begin{gathered}
2 r^{2}(1+r) \frac{\partial^{3} u}{\partial r^{3}}+2 r \frac{\partial^{3} u}{\partial r \partial \theta^{2}}+r(11 r+6) \frac{\partial^{2} u}{\partial r^{2}} \\
+\frac{\partial^{2} u}{\partial \theta^{2}}+\left(2\left(1-\mu^{2}\right)+11 r\right) \frac{\partial u}{\partial r}+u=0 .
\end{gathered}
$$

Lemma 3. Let $0<\operatorname{Re} \mu<1 / 2, g \in L_{1}\left(\mathbb{R}_{+}\right), \beta \in(0,2 \pi)$. Then the function

$$
\begin{aligned}
& u(r, \theta)=\sqrt{\frac{\pi}{1+r}} \int_{0}^{\infty} \Gamma(1+i \tau-\mu) \Gamma(1-i \tau-\mu) \\
& \quad \times P_{i \tau}^{\mu}(\sqrt{1+r}) P_{-i \tau}^{\mu}(\sqrt{1+r}) \frac{\sinh (\theta \tau)}{\sinh (\beta \tau)} g(\tau) d \tau
\end{aligned}
$$

satisfies the partial differential equation (4.2) on the wedge $(r, \theta): r>$ $0,0 \leq \theta<\beta$, vanishing at infinity.

Proof. The proof is straightforward by substitution (4.3) into (4.2) and the use of (1.15). The necessary differentiation with respect to $r$ and $\theta$ under the integral sign is allowed via the absolute and uniform convergence, which can be verified, appealing to the integrability condition $g \in L_{1}\left(\mathbb{R}_{+}\right), \beta \in(0,2 \pi)$ and estimates of derivatives of the kernel (1.3) with respect to $r$. Finally, the condition $u(r, \theta) \rightarrow 0, r \rightarrow \infty$ is due to the estimate of the integral (1.4).

Finally we will formulate the boundary value problem for equation (4.2) and give its solution.

Theorem 5. Let $g(x)$ be given by formula (3.6) and its transform $(G g)(y) \equiv G(y)$ satisfies conditions of Theorem 4 . Then $u(r, \theta), r>$ $0,0 \leq \theta<\beta$ by formula (4.3) will be a solution to the boundary value problem on the wedge for the partial differential equation (4.2) subject to boundary conditions

$$
u(r, 0)=0, \quad u(r, \beta)=G(r) .
$$

\section{Disclosure statement}

No potential conflict of interest was reported by the author.

\section{Funding}

The work was partially supported by CMUP (UID/MAT/00144/2019), which is funded by FCT (Portugal) with national (MEC), European structural funds through the programs FEDER under the partnership agreement PT2020, and Project STRIDE - NORTE-01-0145-FEDER000033, funded by ERDF - NORTE 2020. 


\section{REFERENCES}

1. Yakubovich S. Index transforms. Singapore: World Scientific Publishing Company; 1996.

2. Erdélyi A, Magnus W, Oberhettinger F, Tricomi FG. Higher transcendental functions. Vols. I, II. New York: McGraw-Hill; 1953.

3. Virchenko N, Fedotova I. Generalized associated Legendre functions and their applications (with a Foreword by Semyon Yakubovich). Singapore: World Scientific Publishing Company; 2001.

4. Prudnikov AP, Brychkov YuA, Marichev OI. Integrals and series: Vol. I: Elementary functions. New York: Gordon and Breach; 1986; Vol. II: Special functions. New York: Gordon and Breach; 1986; Vol. III: More special functions. New York: Gordon and Breach; 1990.

5. Yakubovich S, Luchko Yu. The hypergeometric approach to integral transforms and convolutions, Mathematics and its applications. Vol. 287. Dordrecht: Kluwer Academic Publishers Group; 1994.

6. Titchmarsh EC. An introduction to the theory of Fourier integrals. New York: Chelsea; 1986.

7. Lebedev NN. On an integral representation of an arbitrary function in terms of squares of Macdonald functions with imaginary index, Sibirsk. Mat. Zh., 3 (1962), 213-222 (in Russian).

8. Brychkov YuA, Marichev OI, Savischenko NV. Handbook of Mellin transforms. Boca Raton: CRC Press; 2018.

Department of Mathematics, Fac. Sciences of University of Porto, Rua do Campo Alegre, 687; 4169-007 Porto (Portugal) 